\title{
In Silico Tumor Growth: Application to Glioblastomas
}

\author{
Olivier Clatz ${ }^{1}$, Pierre-Yves Bondiau ${ }^{1}$, Hervé Delingette ${ }^{1}$, Grégoire Malandain ${ }^{1}$, \\ Maxime Sermesant ${ }^{1}$, Simon K. Warfield ${ }^{2}$, and Nicholas Ayache ${ }^{1}$ \\ 1 Epidaure - INRIA Sophia Antipolis \\ 2 CRL - Harvard Medical School
}

\begin{abstract}
We propose a new model to simulate the growth of glioblastomas multiforma (GBM), the most aggressive glial tumors. This model relies upon an anatomical atlas including white fibers diffusion tensor information and the delineation of cerebral structures having a distinct response to the tumor aggression. We simulate both the invasion of the GBM in the brain parenchyma and its mechanical interaction (mass effect) with the invaded structures. The former effect is modeled with a reaction-diffusion equation while the latter is based on a linear elastic brain constitutive equation. In addition, we propose a new equation taking into account the mechanical influence of the tumor cells on the invaded tissues. This tumor growth model is assessed by comparing the virtual GBM growth with the real GBM growth observed between two MRIs of a patient acquired with six months difference.
\end{abstract}

\section{Introduction}

\subsection{Motivation}

The majority of the primitive tumors of the central nervous system are from glial origin, among which the glioblastomas multiforma (GBM) are the most aggressive. Without therapy, patients with GBMs usually die within 10 months. Despite the substantial research effort for improving tumors treatment, patients treated with state-of-the-art therapy have a median survival of approximately 1.5 year.

Relatively little progress has been made toward the construction of a general model describing the growth of these tumors. The interest to carry out a simulation of the tumoral growth for improving the treatment is twofold. First, it could provide additional information about the tumor invasion and help determining the local treatment margins. Second, by quantifying the malignant cell concentration in low contrast areas of MR images, it could also be useful in the selection of the radiotherapy dose.

\subsection{Contributions}

We propose a patient-specific simulator of glioblastoma growth, including the induced brain deformation (mass effect). The simulation relies upon a Finite 
Element Model (FEM) initialized from the patient MRIs. Additional information has been included into the patient model to take into account the behavior of different structures with respect to tumor invasion, such as the white matter fiber directions (see [2] for details about the atlas construction). Furthermore, we propose to link the classification of tumors in Gross Tumor Volumes (GTV) proposed in protocols for radiotherapy treatment with different tumor invasion behaviour:

- the GTV1 is associated with the expansion component. By creating new cells, the GTV1 pushes away its surrounding structures. It is therefore responsible for the major mechanical mass effect on the brain. Following cellular expansion models, we propose to use an exponential law to describe the GTV1 volume increase.

- The GTV2 is associated with the diffusion component. It invades adjacent structures by a diffusion process. The GTV2 is thus described in our model with a reaction-diffusion equation. In addition, we propose to link the diffusion process to the mechanical mass effect with a coupling equation.

The model is initialized from an early patient MRI and the simulation is compared to the patient MR images acquired six months later. Compared to

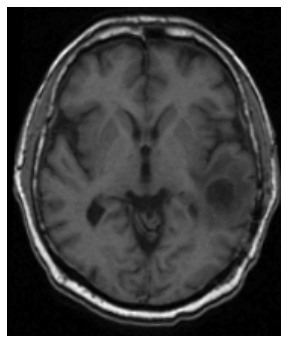

(a)

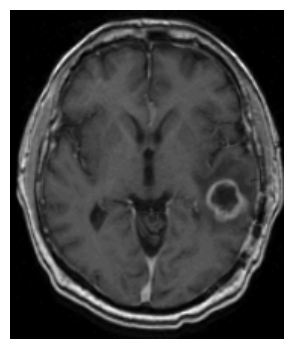

(b)

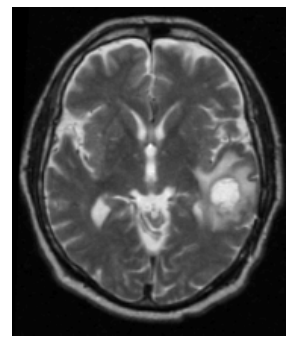

(c)

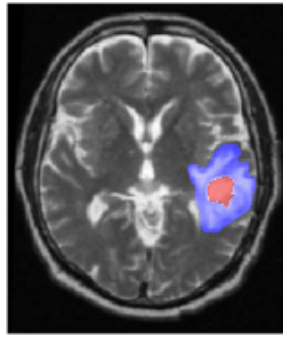

(d)

Fig. 1. MR images of a patient (a) T1; (b) T1 with gadolinium injection; (c) T2; (d) GTV1 (red) and GTV2 (blue) segmentations overlaid on the T2 MRI.

previous works in the tumor growth modeling domain ([7/834]), our approach includes several improvements:

- the use of diffusion tensor imaging to take into account the anisotropic diffusion process in white fibers.

- The use of the radiotherapy volume classifications to initialize the source of the diffusion component (as opposed to point sources in [7]).

- A new coupling equation between the reaction-diffusion equation and the mechanical constitutive equation.

- Initialization with a patient tumor and comparison with the invasion observed into the later patient MR images. 


\section{Material and Methods}

Our GBM growth simulation consists of two coupled models:

1. a model for the diffusion of the tumor that captures the evolution of the tumor density $c$ over time.

2. A model for the expansion of the tumor that predicts the mass effect induced by the tumor proliferation.

The coupling between these two models is further described in section 3 but it assumes the following behavior : the mass effect is directly related to the tumor density $c$ but the tumor density $c$ is not influenced by the mass effect.

This simple coupling leads to a four steps algorithm:

- Image segmentation and registration. The two gross volumes GTV1 and GTV2, are manually delineated by an expert from the patient MR images. The patient MR images are registered with respect to an anatomical atlas. This atlas includes for each voxel the location of the main cerebral structures and a diffusion tensor in the white matter.

- Meshing and Initialization. A tetrahedral mesh of the patient's brain is built in the atlas reference frame. Tissue properties are assigned to their associated tetrahedra using the atlas. Furthermore, the value of the tumor density $c$ is initialized based on the GTV1 and GTV2 segmentations by interpolating between the two boundaries.

- Simulation. The simulation of the VG (Virtual Glioblastoma) diffusion and expansion is performed on the finite element mesh following the mechanical and diffusion equations.

- Comparison. At the end of the growth process, new GTV1, GTV2 and local deformations of the atlas are reported back in the patient images. Therefore, an assessment of the model is performed by comparing the predicted tumor volumes with the ones observed from patient MR image acquired six months later.

\section{Glioblastoma Growth Simulation}

\subsection{Diffusion Model}

We rely on the classical reaction-diffusion model ([7]) to account for the growth and the spreading of tumor cells in the GTV2:

$$
\underbrace{\frac{\partial c}{\partial t}}_{\text {Tumor density evolution }}=\underbrace{\operatorname{div}(\underline{\underline{D}} \underline{\nabla c})}_{\text {Diffusion law }}+\underbrace{S(c, t)}_{\text {Source factor }}-\underbrace{T(c, t)}_{\text {Treatment law }}
$$

In this equation, $c$ represents the normalized cell density $(c \in[0,1])$. The real cell density $C$ is obtained by multiplying $c$ with the carrying capacity of the

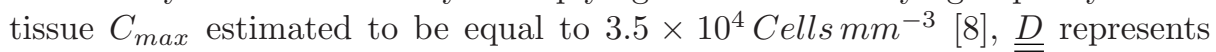


the local diffusivity of the tissue and depends on the nature of the tissue or, for white matter, of the white fiber directions. Since the goal is only to simulate the tumor growth, we do not consider the treatment term $T(c, t)$ for this model. To minimize the number of tumor-intrinsic parameters, we use a simple linear function to model the source factor, reflecting its aggressiveness: $S(c, t)=\rho c$. The diffusion law 1 can then be written as:

$$
\frac{\partial c}{\partial t}=\operatorname{div}(\underline{\underline{D}} \underline{\nabla c})+\rho c
$$

The local behavior of the tumor therefore only depends on the diffusion tensor $\underline{\underline{D}}$ and the source factor $\rho$.

Model Parameters and Initialization. We propose the following characteristics for the model:

- since the conductivity of skull and ventricles is null, the flux at the mesh surface is zero: $\underline{J} \cdot \boldsymbol{n}=0$

- We use the diffusion tensor of the atlas to initialize the diffusion tensor $\underline{D}$ in white matter. The intrinsic aggressiveness of the tumor is then controlled by two parameters $\alpha$ and $\beta$.

- There are several evidences that glioblastomas diffuse more slowly in the gray matter than in the white matter [7]. Thus diffusivity in gray matter is chosen as a fraction of the maximum diffusivity in white matter $\beta=\frac{D_{\text {white }}}{D_{\text {gray }}}=\frac{1}{100}$.

- Because tumor cells cannot diffuse through the falx cerebri, we set its diffusivity to zero.

- The GTV1 capacity is fixed to $C_{\max }$ as above-defined.

- As discussed in [7, one cannot determine both $\alpha$ and $\rho$ from only two different instants. We thus arbitrarily set $\rho=\frac{\eta}{100}(\eta$ is defined in section 3.2 ). The $\alpha$ parameter is then adapted to the GTV2 diffusion speed.

The material diffusivity values are summed up in Table 1. Figure 2 summarizes the diffusion model and the boundary conditions. We use the model of equation 2 to solve the stationary problem, so as to interpolate the $c$ function between the two initial contours delineating the GTV1 and GTV2.

\subsection{Mechanical Model}

Mechanical Equation. Based on rheological experiments, Miller ([5]) proposed a non-linear constitutive equation for very slow deformations. Since the growing process is extremely slow in our case, and the measured deformation in the parenchyma is in the small deformation range $(\leq 5 \%)$, we propose to linearize this equation. Choosing the Young modulus $E=694 \mathrm{~Pa}$, the absolute stress error committed on the stress with respect to Miller's model is below 4.2 $\mathrm{Pa}$ (see [2] for details). We thus consider linear relationship for both the constitutive equation and the strain computation:

$$
\underline{\underline{\sigma}}=\underline{\underline{K}} \underline{\underline{\epsilon}} \quad \text { and } \quad \underline{\underline{\epsilon}}=\frac{1}{2}(\underline{\underline{\nabla u}}+\underline{\underline{\nabla u}})
$$


$-\underline{\underline{K}}$ is the rigidity matrix $(\mathrm{Pa})$.

$-\underline{\bar{\sigma}}$ the internal stress tensor $(\mathrm{Pa})$

- $\underline{\underline{\epsilon}}$ is the linearized Lagrange strain tensor expressed as a function of the displacement $\underline{u}$ (no unit).

Table 1. Stiffness and diffusivity properties of the finite element model

\begin{tabular}{|l|c|c|c|}
\hline Tissue & $\begin{array}{c}\text { Young } \\
\text { Modulus }(\mathrm{Pa})\end{array}$ & $\begin{array}{c}\text { Poisson } \\
\text { Coefficient }\end{array}$ & $\begin{array}{c}\text { Tissue diffusivity } \\
\left(10^{-3} \mathrm{~mm}^{2} \mathrm{~s}^{-1}\right)\end{array}$ \\
\hline White Matter & 694 & 0.4 & $\alpha \cdot$ DTI (anisotropic) \\
\hline Gray Matter & 694 & 0.4 & $\beta \cdot \max (\underline{\underline{D}}($ White $))$ \\
\hline Falx Cerebri & 200.000 & 0.4 & 0 \\
\hline Ventricles & 0 & 0 & 0 \\
\hline Skull & $\infty$ & 0.5 & 0 \\
\hline
\end{tabular}

Because the GTV1 is modeled as a pure cell proliferation and since the associated tissue is already considered as saturated, this proliferation directly acts as a volume increase $\Delta V$ on the GTV1:

$$
\Delta V=V_{t}-V_{0}=V_{0}\left(e^{\eta t}-1\right)
$$

Based on the proposed model, $\eta$ can be approximated by computing the average volume increase of GTV1 in GBM. We found $\eta=2.2 \times 10^{-3}$ day $^{-1}$. We use a penalty method to impose this volume variation boundary condition via a homogeneous pressure force into the GTV1.

We propose a new equilibrium equation to model the mechanical impact of the tumor on the invaded structures.

$$
\operatorname{div}\left(\underline{\underline{\sigma}}-\lambda c \underline{\underline{\mathbb{I}_{3}}}\right)+\underline{\underline{f_{\text {ext }}}}=0
$$

This equation is the differential version of the law proposed by Wasserman 9 . It can be locally interpreted as a tissue internal pressure $\lambda c$ proportional to the tumor concentration $c$. This law is used to describe the mechanical effects of the malignant cells invading the brain parenchyma.

Model Parameters and Initialization. The proposed mechanical model is similar to the one used for predicting intra-operative deformations [1]. It has the following characteristics:

- the skull does not deform and is considered as beeing infinitely rigid. Thus vertices on the surface of the brain mesh are fixed.

- We use the linearized 3D homogeneous version of Miller's constitutive equation (see 3.2 for details), the Young modulus is set to $694 \mathrm{~Pa}$ and the Poisson coefficient is thus set to 0.40 . 

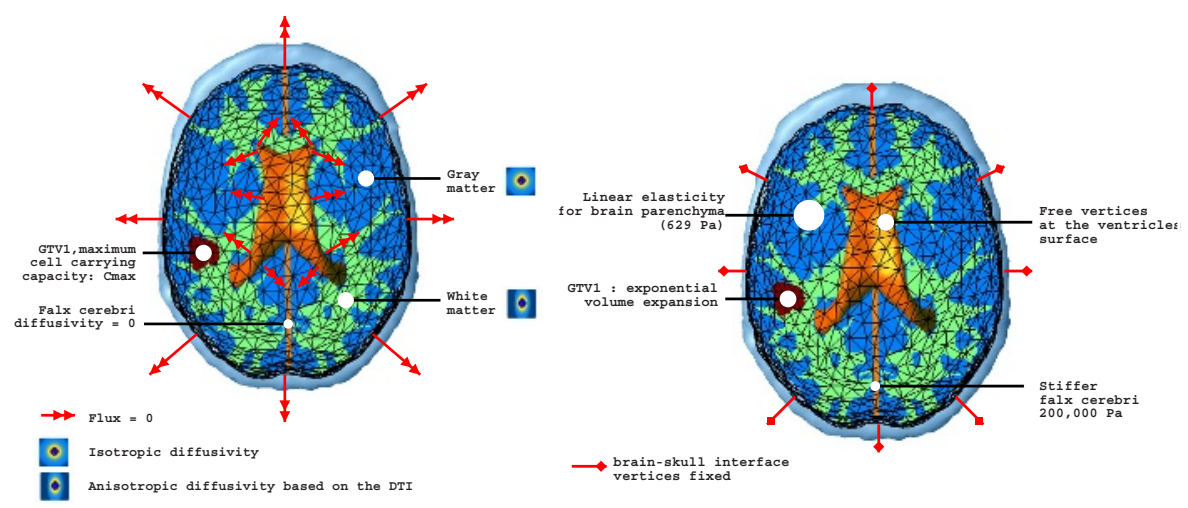

Fig. 2. Summary of the diffusion (left) and the mechanical (right) model.

- We consider that the ventricular pressure is not affected by the tumor growth. Therefore we let ventricular vertices free without additional internal pressure.

- Based on the rheological experiments [6] made on the falx cerebri, we choose its Young modulus equal to $2 \times 10^{5} \mathrm{~Pa}$.

- We choose a coupling factor $\lambda$ which minimizes the quantitative difference between the model and the real deformations: $\lambda=1.4 \times 10^{-9} \mathrm{~N} \mathrm{~mm} \mathrm{Cells}^{-1}$.

The material mechanical properties are summed up in Table 1 Figure 2 summarizes the diffusion model and the boundary conditions.

\section{Results}

After performing the simulation, we registered both the deformations and the tumor concentration into the first patient MRI (03/2001). Results are presented in two parts, the mass effect and the tumor diffusion.

Mass Effect. Figure 3 shows the displacement of internal tissue due to the mass effect. Even if this major displacements take place close to the GTV1, further away tissues in the same hemisphere are also affected by the tumor growth. The average displacement at the GTV1-GTV2 frontier is $3 \mathrm{~mm}$. The tumor has an influence on the lateral ventricles size (volume variation $\Delta V=$ $4.6 \mathrm{ml}$ ). To quantify the accuracy of the simulation, a medical expert manually selected corresponding feature points on the patient MRIs so as to estimate these landmark displacements between March 2001 and September 2001. These measured displacements can then be compared to the one simulated by the model (complete landmark positions and errors can be found in [2]). The average displacement for selected landmarks is $2.7 \mathrm{~mm}$ and the corresponding average error is $1.3 \mathrm{~mm}$. Without recovering the entire deformation, the proposed model captures the largest part of the displacement. The remaining error might be due to different phenomena: 

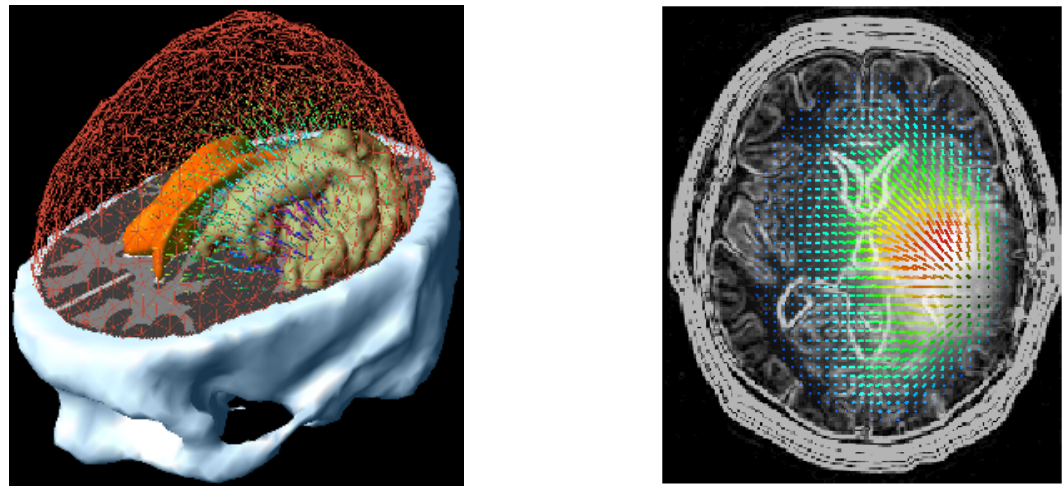

Fig. 3. Displacement of the tissues induced by the tumor mass effect.

- the ratio between the average deformation amplitude $(2.7 \mathrm{~mm})$ and the image resolution $(1.0 \mathrm{~mm})$ is in the range of manual selection error.

- The deformation phenomenon might be larger in interstitial space than in the brain parenchyma. In such a case, a finer mesh and different constitutive equations would be necessary to model the deformation.

Diffusion. Results on two different axial slices of the diffusion process can be seen on figure 4 The four columns should be read as follow:

- column 1 shows the T2 weighted MRI acquired in March 2001.

- Column 2 shows the same MRI with interpolated contours used to initialize the model.

- Column 3 shows the T2 weighted MRI of the same patient acquired 6 months later.

- Column 4 shows the same MRI with simulated tumor isodensity curves.

\section{Perspectives}

Model Improvement for Simulation. Previous results have demonstrated the ability of the numerical model to predict the tumor behavior. However, the model could be enhanced with additional characteristics:

- the modification of the fiber structures in the invaded area.

- The model could largely benefit from the use of more patient-specific images. More precisely, patient DTI capturing the white-matter fiber directions could greatly improve the accuracy of the simulation.

Clinical Validation and Applications. We consider the comparison of the simulated VG with the follow-up MR image of the patient as a preliminary step for validating the proposed model. We wish to develop other methods for the identification of parameters and for clinical validation: 
- correlation of the VG prediction with histopathological analysis of patient brains, especially in the MRI areas under the threshold of detection.

- Adding functional information into the atlas to allow the prediction of functional loss induced by the tumor growth.
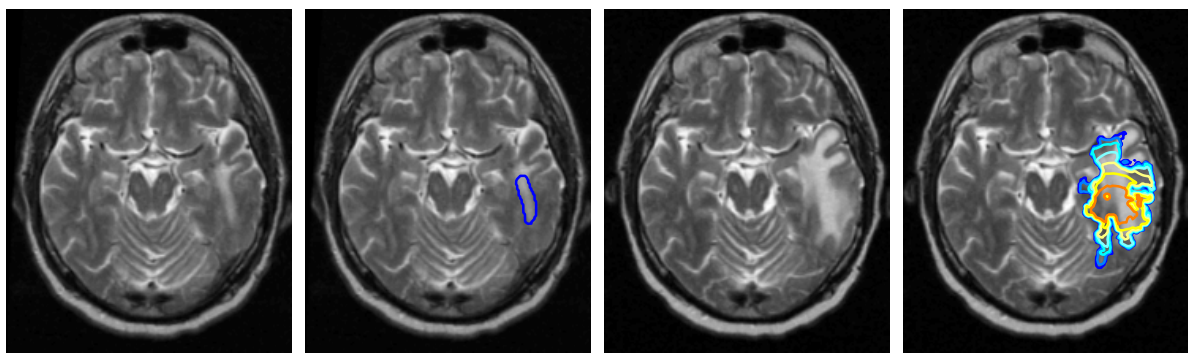

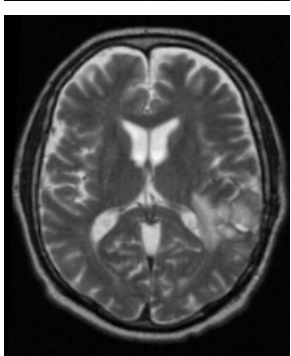

1

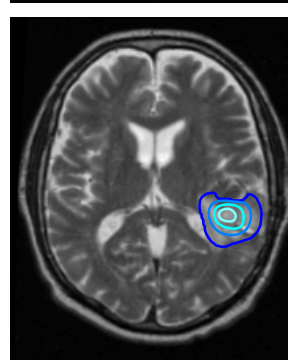

2

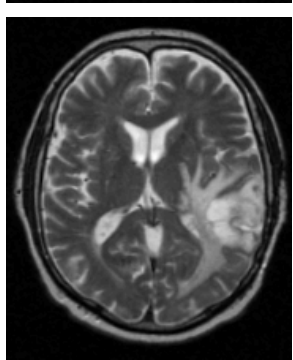

3

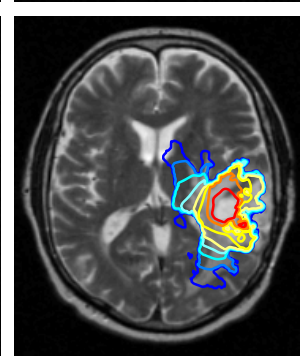

4

Fig. 4. 1. T2 MRI 03/2001, 2. T2 MRI 03/2001 + GBM initialization, 3. T2 MRI 09/2001, 4. T2 MRI 09/2001 + simulated GBM tumor isodensities

\section{References}

1. O. Clatz, H. Delingette, E. Bardinet, D. Dormont, and N. Ayache. Patient specific biomechanical model of the brain: Application to parkinson's disease procedure. In N. Ayache and H. Delingette, editors, International Symposium on Surgery Simulation and Soft Tissue Modeling (IS4TM'03), volume 2673 of LNCS, pages 321-331, Juan-les-Pins, France, 2003. INRIA Sophia Antipolis, Springer-Verlag.

2. O. Clatz, P.Y. Bondiau, H. Delingette, M. Sermesant, S.K. Warfield, G. Malandain, and N. Ayache. Brain tumor growth simulation. Research report 5187, INRIA, 2004.

3. B. M. Dawant, S. L. Hartmann, and S. Gadamsetty. Brain atlas deformation in the presence of large space-occupying tumors. Medical Image Computing and ComputerAssisted Intervention, volume 1679 of LNCS, pages 589-596, 1999.

4. S. K. Kyriacou and C. Davatzikos. A biomechanical model of soft tissue deformation, with applications to non-rigid registration of brain images with tumor pathology. Proceedings of the First International Conference on Medical Image Computing and Computer-Assisted Intervention, volume 1496 of LNCS, pages 531-538, 1998.

5. K. Miller. Biomechanics of Brain for Computer Integrated Surgery. Warsaw University of Technology Publishing House, 2002. ISBN:83-7207-347-3.

6. M. Schill, M. Schinkmann, H.-J. Bender, and R. Männer. Biomechanical simulation of the falx cerebri using the finite element method. In 18. Annual International Conference, IEEE Engeneering in Medicine and Biology, 1996. 
7. K.R. Swanson, E.C. Alvord Jr, and J.D. Murray. Virtual brain tumours (gliomas) enhance the reality of medical imaging and highlight inadequacies of current therapy. British Journal of Cancer, 86(1):14-18, Jan 2002.

8. P. Tracqui. From passive diffusion to active cellular migration in mathematical models of tumour invasion. Acta Biotheoretica, 43(4):443-464, Dec 1995.

9. R. Wasserman and R. Acharya. A patient-specific in vivo tumor model. Mathematical Biosciences, 136(2):111-140, Sep 1996. 